\title{
ON A DISTRIBUTION OF LEIPNIK AND PEARCE
}

\author{
SARALEES NADARAJAH ${ }^{\circ 1}$ and SAMUEL KOTZ ${ }^{2}$ \\ We dedicate this note to the memory and kindness of Professor Roy B. Leipnik.
}

(Received 9 May, 2006)

\begin{abstract}
A recent paper by Leipnik and Pearce introduced the gamma Weibull distribution. One of the main properties given is its characteristic function, which is expressed as an infinite sum. In this note, we provide a simpler representation in terms of the well-known hypergeometric functions in some special cases. We also derive expressions for moments of the distribution.

2000 Mathematics subject classification: primary 33C90; secondary 62E99.

Keywords and phrases: gamma distribution, Weibull distribution.
\end{abstract}

\section{Introduction}

By multiplying the gamma and Weibull densities and renormalizing, Leipnik and Pearce [2] introduced a novel distribution referred to as the gamma Weibull distribution. The probability density function (pdf) of this distribution takes the form

$$
f(x)=K x^{\alpha-1} \exp \left(-\mu x-a x^{r}\right)
$$

for $x>0, r>0, a>0, \mu>0$ and $\alpha>0$, where $K=K(r, a, \mu, \alpha)$ is the normalizing constant. Note that the corresponding form of the pdf given in Equation (2.1) of Leipnik and Pearce[2] has five parameters; in fact, only four parameters are needed to define the gamma Weibull distribution.

Leipnik and Pearce derived the characteristic function (chf) and discussed sums of independent random variables having the gamma Weibull distribution. Every property

\footnotetext{
${ }^{1}$ School of Mathematics, University of Manchester, Manchester M60 1QD, UK; e-mail: saralees.nadarajah@manchester.ac.uk.

${ }^{2}$ Department of Engineering Management and Systems Engineering, George Washington University, Washington, DC 20052, USA.
}

(C) Australian Mathematical Society 2007, Serial-fee code 1446-1811/07 
derived in the paper is expressed as infinite sums. In particular, the chf is expressed as an infinite sum of gamma functions. In this note, we show that when $r$ is rational a simpler form can be derived in terms of the well-known hypergeometric function defined by

$$
{ }_{p} F_{q}\left(a_{1}, \ldots, a_{p} ; b_{1}, \ldots, b_{q} ; x\right)=\sum_{k=0}^{\infty} \frac{\left(a_{1}\right)_{k}\left(a_{2}\right)_{k} \cdots\left(a_{p}\right)_{k}}{\left(b_{1}\right)_{k}\left(b_{2}\right)_{k} \cdots\left(b_{q}\right)_{k}} \frac{x^{k}}{k !},
$$

where $(f)_{k}=f(f+1) \cdots(f+k-1)$ denotes the ascending factorial. We also derive simple forms for the moments associated with (1.1) - a property not discussed by Leipnik and Pearce.

\section{Characteristic function}

Let $X$ be a random variable with pdf given by (1.1). The chf of $X$ can be expressed as

$$
\begin{aligned}
\phi(t) & =E[\exp (i t X)] \\
& =K \int_{0}^{\infty} x^{\alpha-1} \exp \left(i t x-\mu x-a x^{r}\right) d x \\
& =K \int_{0}^{\infty} x^{\alpha-1} \exp \left(-s x-a x^{r}\right) d x \\
& =K I(\alpha, s, a, r),
\end{aligned}
$$

where $s=\mu-$ it. By Equation (2.3.1.13) in Prudnikov et al. [3, Volume 1], the integral $l$ in (2.1) can be evaluated as

$$
I=\left\{\begin{array}{l}
\sum_{j=0}^{q-1}{\frac{(-a)^{j} \Gamma(\alpha+r j)}{j ! s^{\alpha+r j}}}_{p+1} F_{q}\left(1, \Delta(p, \alpha+r j) ; \Delta(q, 1+j) ;(-1)^{q} z\right), \\
\quad \text { if } 0<r<1, \\
\sum_{h=0}^{p-1} \frac{(-1)^{h} s^{h}}{r h ! a^{(\alpha+h) / r}} \Gamma\left(\frac{\alpha+h}{r}\right) \\
\times_{q+1} F_{p}\left(1, \Delta\left(q, \frac{\alpha+h}{r}\right) ; \Delta(p, 1+h) ; \frac{(-1)^{p}}{z}\right), \quad \text { if } r>1, \\
\frac{1}{(a+s)^{\alpha}}, \quad \text { if } r=1,
\end{array}\right.
$$

provided that $r=p / q$, where $p \geq 1$ and $q \geq 1$ are co-prime integers, where $z=p^{p} a^{q} /\left\{s^{p} q^{q}\right\}$ and $\Delta(k, a)=(a / k,(a+1) / k, \ldots,(a+k-1) / k)$. Thus, the chf 
of $X$ can be expressed as finite sums of the hypergeometric function provided that $r$ is a rational number. The hypergeometric functions are well known and well established (see [1, Section 9.14]). Numerical routines for computing them are widely available (see for example Maple and Mathematica).

\section{Moments}

Similar expressions can be obtained for the moments. If $X$ is a random variable with pdf given by (1.1) then its $n$th moment can be expressed as

$$
\begin{aligned}
E\left(X^{n}\right) & =K \int_{0}^{\infty} x^{n+\alpha-1} \exp \left(-\mu x-a x^{r}\right) d x \\
& =K I(n+\alpha, \mu, a, r),
\end{aligned}
$$

where $I$ is given by (2.2). In particular, the normalizing constant $K$ is given by $K=1 / I(\alpha, \mu, a, r)$.

\section{References}

[1] I. S. Gradshteyn and I. M. Ryzhik, Table of Integrals, Series, and Products, 6th ed. (Academic Press, San Diego, CA, 2000).

[2] R. B. Leipnik and C. E. M. Pearce, "Independent non-identical five-parameter gamma-Weibull variates and their sums", ANZIAM J. 46 (2004) 265-271.

[3] A. P. Prudnikov, Y. A. Brychkov and O. I. Marichev, Integrals and Series, Volumes 1, 2 and 3 (Gordon and Breach Science Publishers, Amsterdam, 1986). 